\title{
A 48-year-old man with chronic right shoulder pain and weakness after a fall
}

\author{
Matthew D. Epstein • Puneet Bhargava • \\ Jonathan R. Medverd
}

Published online: 30 January 2010

(C) ISS 2010

\section{History}

A 48-year-old man presented to the orthopedic surgery clinic for evaluation of significant right shoulder pain, weakness, and numbness on the anterior aspect of his right shoulder. Eleven months previously, the patient fell off a ladder and sustained an anterior right shoulder dislocation. The patient was able to reduce his shoulder himself within 5 min. MRI of the shoulder was performed (Fig. 1).

The diagnosis can be found at doi:10.1007/s00256-009-0841-4.

M. D. Epstein

Department of Radiology, University of Washington,

1959 NE Pacific Street, Box 357115, Seattle, WA 98195-7115,

USA

e-mail: mde1@u.washington.edu

P. Bhargava $(\square) \cdot J$. R. Medverd

Department of Radiology, University of Washington \& VA Puget

Sound Health Care System,

1660 S Columbian Way, S-114/Radiology, Mail Stop 358280,

Seattle, WA 98108 , USA

e-mail: bhargp@u.washington.edu

J. R. Medverd

e-mail: jmed@u.washington.edu 
Fig. 1 a Proton density $(P D$; repetition time $\mathrm{ms} / \mathrm{echo}$ time $\mathrm{ms}$, $1,618 / 20)$ sequence coronal oblique image; b T2-weighted spectral presaturation with inversion recovery (SPIR; repetition time $\mathrm{ms} / \mathrm{echo}$ time $\mathrm{ms}$, $3,142 / 60$ ) sequence coronal oblique image; c, d T2-weighted (repetition time ms/echo time ms, 1,542/62) sequence sagittal oblique images. Increased signal and fatty atrophy is seen in the deltoid (white arrows) and the teres minor (black arrows). Sup supraspinatus muscle, $I$ infraspinatus muscle, Sub subscapularis muscle
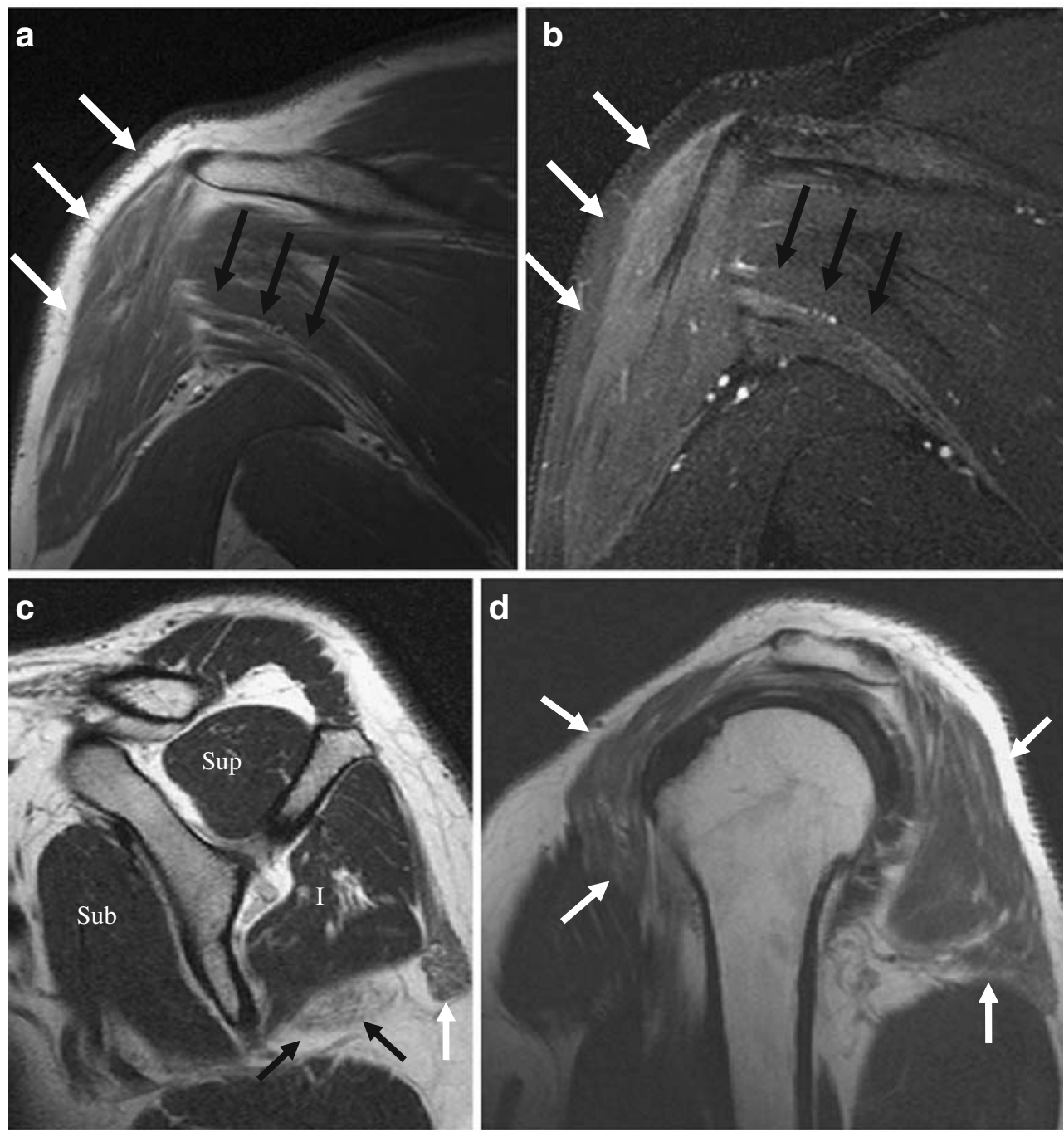

c 